\title{
Total Screening of Blood Donations for Australia (Hepatitis Associated) Antigen and its Antibody
}

\author{
J. WALLACE, G. R. MILNE, A. BARR
}

British Medical fournal, 1972, 1, 663-664

\section{Summary}

During a period of one year all of 105,724 blood donations were tested for Australia ( $\mathrm{Au}$ ) antigen and its antibody by rapid immunoelectro-osmophoresis-86 (1 in 1,229) were positive for antigen and $67(1$ in 1,578$)$ positive for antibody. Second donations by previously negative donors reduce the overall incidence of positives. Men prisoners have a significantly higher incidence of Au antigen ( 1 in 153) than non-institutionalized men (1 in 803$)$. The latter have a significantly higher incidence of antigen than women (1 in 2,019). Only one antigen-positive donor was incubating acute viral hepatitis. Failure to detect one strong and one weak antigen was responsible for two cases of posttransfusion Au-antigen-positive hepatitis.

\section{Introduction}

The Bulletin of the World Health Organization (1970) recommended the detection and exclusion of blood donors carrying Australia $(\mathrm{Au})$ antigen. Adoption of this recommendation should make transfusion therapy safer and lead to a better understanding of the epidemiology of serum hepatitis. The presence of antibody to Au antigen is presumptive evidence of previous exposure to the infective agent. In the present state of knowledge it is advisable to detect and exclude donors with antibody. The finding of antibody will provide testing reagents and also plasma for the production of specific anti-Au antigen IgG immunoglobulin.

This regional transfusion service performed partial screening of donations for Au antigen and its antibody for a period of six months (Wallace, 1970), and then introduced total screening (Milne et al., 1971). The results of one year of total screening are now reported.

\section{Material and Methods}

Between October 1970 and October 1971 serum from 105,724 donations was tested for $\mathrm{Au}$ antigen and its antibody by the method of immunoelectro-osmophoresis as modified by Milne and Barr (1971). Testing reagents of human origin were used. Screening tests were performed on the day after donation, being completed by 1 p.m. The largest number of donations tested in one morning was 650 , the normal range being 350 to 450 . All sera positive for $\mathrm{Au}$ antigen in the screening test (whether convincing or equivocal) were checked against eight antisera known to give specific reactions for $\mathrm{Au}$ antigen. Six of these antisera, including the screening antiserum, were of human origin. The remaining two antisera were of animal origin. Seven sera known to be negative for $\mathrm{Au}$ antigen were tested at the same time as negative controls. A second confirmatory test for Au antigen was performed with a conventional complement fixation technique. Here only one antiserum of animal origin was used.

Glasgow and West of Scotland Regional Transfusion Centre, Law Hospital, Carluke, Lanarkshire ML8 5ES

J. WALLACE, M.D., F.R.C.P., Regional Director G. R MILNE, F.P.S., Deputy Regional Director A. BARR, F.I.M.L.T., Chief Technician

Sera positive for antibody in the screening tests were checked against 15 known sera, of which eight were positive and seven negative for $\mathrm{Au}$ antigen.

Some donors had been tested previously, during the period of partial screening. Others donated more than once during the year. For the purpose of analysis the total donations are divided into 31,884 previously tested and 73,840 tested for the first time. The respective incidences of antigen and antibody were studied in men and women, and in men donors from H.M. Prisons.

\section{Results}

Table I shows that 86 donors were Au-antigen positive and another 67 antibody positive. Of these 153 positive donors, 149 were being tested for the first time. One antigen-positive and three antibody-positive donors had been found negative at a previous donation. The significance of this is discussed below. None of the positive donors admitted, at the time of donation, to having had an illness characterized by jaundice, or to having received a blood transfusion. On subsequent questioning four admitted to having had an illness several years ago which might have been hepatitis. All these positive donors have remained well with the exception of an 18-year-old nurse who felt unwell 10 days after donation, and developed hepatitis. She made a good recovery, the antigen level in the blood falling to zero within two months.

Table II shows the incidence of antigen and antibody at the first time of testing men prisoners and non-institutionalized men and women. The higher incidence of $\mathrm{Au}$ antigen in prisoners compared with other men is highly significant $\left(\chi^{2}=31.062, \mathrm{P}<1 \times 10^{-7}\right)$. Similarly the higher incidence of Au antigen in non-institutionalized men compared with women is statistically significant $\left(\chi^{2}=7.794,0.01>P>0.005\right)$. Although there is a higher incidence of antibody in men prisoners and in women than in non-institutionalized men, these differences are not statistically significant.

\begin{tabular}{|c|c|c|c|c|}
\hline & & $\begin{array}{l}\text { Donor Tested } \\
\text { for First Time }\end{array}$ & $\mid \begin{array}{c}\text { Donor Pre- } \\
\text { viously Negative }\end{array}$ & Total \\
\hline $\begin{array}{l}\text { Number } \\
\text { Au-antigen positive } \\
\text { Au-antibody positive }\end{array}$ & $\begin{array}{l}\cdots \\
\cdots\end{array}$ & $\begin{array}{l}73,840 \\
85(1: 869) \\
64(1: 1,154)\end{array}$ & $\begin{array}{c}31,884 \\
1(1: 31,884) \\
3(1: 10,628)\end{array}$ & $\begin{array}{c}105,724 \\
86(1: 1,229) \\
67(1: 1,578)\end{array}$ \\
\hline
\end{tabular}

TABLE II-Incidence of Australia Antigen and Antibody in Donors Tested for First Time

\begin{tabular}{|c|c|c|c|c|}
\hline & & \multicolumn{2}{|c|}{ Males } & \multirow{2}{*}{ Females } \\
\hline & & Institutionalized & $\begin{array}{c}\text { Not } \\
\text { Institutionalized }\end{array}$ & \\
\hline $\begin{array}{l}\text { Number } \\
\text { Au-antigen positive } \\
\text { Au-antibody positive }\end{array}$ & $\begin{array}{l}. \\
\therefore\end{array}$ & $\begin{array}{c}1,835 \\
12(1: 153) \\
3(1: 612)\end{array}$ & $\begin{array}{c}49,798 \\
62(1: 803) \\
39(1: 1,277)\end{array}$ & $\begin{array}{c}22,207 \\
11(1: 2,019) \\
22(1: 1,009)\end{array}$ \\
\hline
\end{tabular}

\section{Discussion}

While the Bulletin of the World Health Organization (1970) advocated total screening of donations, it emphasized that the application of the present relatively insensitive tests may reduce the risk to recipients by less than $25 \%$. Since serum hepatitis may have an incubation period as long as six months, it is too early to assess the full significance of total screening in the 
present survey. However, the occurrence of two cases of posttransfusion Au-antigen-positive hepatitis does show that the present screening method will not detect all carriers.

The first patient had received blood from eight donors. During investigation it was noted that a weakly positive reactor for $\mathrm{Au}$ antigen on the screening test was one of the eight donors involved. The retesting of the other donors has given negative reactions for antigen and antibody. It seems likely that this one donor, shown in Table I as the only Au-antigen positive among donors previously negative, was responsible for transmitting the disease. Serum from the earlier, apparently negative donation had not been preserved. Fortunately it was possible to obtain a specimen of serum taken from the same individual for another purpose 18 months earlier. This earlier specimen and the recent specimen gave a weak positive reaction for $\mathrm{Au}$ antigen on the routine screening test. Serial dilutions of the donor's serum showed a marked prozone phenomenon by immunodiffusion and complement fixation as well as by immunoelectroosmophoresis.

The second case of posttransfusion Au-positive hepatitis illustrates the converse problem of detecting weak antigens in donor sera. The recipient had been given 23 donations. The donors were retested and one gave a weakly positive reaction by immunoelectro-osmophoresis and by complement fixation, though negative by immunodiffusion. Electron microscopy of this serum showed a few characteristic clumps after the addition of anti-Au serum. Again the original specimen of the donor serum was not available. All donor sera are now being preserved for at least six months after testing.

Immunoelectro-osmophoresis is an attractive technique for screening donations, because answers can be obtained so rapidly (Cossart, 1971). False negatives may occur if there is an excess of antigen or of antibody (Kohn and Morgan, 1971). This happened in the above two donations which almost certainly transmitted Au-antigen-positive hepatitis-one donor had a strong antigen and the other a weak antigen. The strong antigen was certainly detectable by complement fixation, immunoelectro-osmophoresis, and immunodiffusion provided a range of dilutions of antigen was used, but the weak antigen was barely detectable by immunoelectro-osmophoresis and complement fixation, and could be confirmed only by immune electron microscopy. In choosing a method for testing donations it is helpful to know the concentration of antigen or antibody likely to be encountered. The selection of antisera for this study has been based on the ability to detect Au antigen in the concentrations so far found in donors. Time will show whether or not the two antigens missed at the original screening represent the extreme ends of the range of concentration in apparently healthy donors, and how often such concentrations occur.

An immediate concern on finding an Au-positive donor is that the individual may be incubating viral hepatitis. No donor so far has refused to allow a report to be sent to the general practitioner. In only one case, the 18 -year-old nurse mentioned above, has an Au-antigen-positive donor developed overt hepatitis. It is interesting to speculate what might have happened if this donor had volunteered one to two weeks earlier. It seems possible that the antigen might not have been detected, though the infective agent might have been present in the blood. This surely represents a dangerous donor in relation to the transmission of disease, but the frequency with which such a donor might be encountered is uncertain.

This one donor who developed acute hepatitis is the only Au-antigen-positive donor known to have reverted to antigen negative. Of the others 28 antigen and 28 antibody positives have been retested at intervals varying from 2 to 12 months after the initial positive test, and all have remained positive. Four of the antigen-positive donors had come under suspicion before the discovery of the association between $\mathrm{Au}$ antigen and hepatitis, because of being among donors involved in cases of posttransfusion hepatitis. These cases of hepatitis occurred three and four years earlier. The follow-up of the recipients of previous donations of Au-antigen-positive donors has proved time-consuming and frustrating, because of the inadequacy of some hospital records. Although these antigen-positive and antibody-positive donors seem healthy, a detailed study of liver function is being conducted.

\section{INCIDENCE}

Population surveys show considerable geographical variation in the incidence of $\mathrm{Au}$ antigen in blood donors (Cossart, 1971). The incidence of 1 in $869(0.115 \%)$ shown in Table I for donors tested for the first time is in agreement with the range of about $0.1 \%$ for unpaid donors in the U.S.A. and Western Europe. The information about the retesting of previously negative donors shown in Table $I$ suggests that negative donors are unlikely to become positive within the next 6 to 12 months. The one Au-antigen-positive donor in the previously tested group exhibited a prozone, and probably for that reason had been missed at the earlier test. The three antibodies among the previously negative donors were weak, and may well have been missed at the original test. These three donors gave no history of recent illness, and the earlier donations did not cause overt hepatitis in the recipients.

The high incidence of $\mathrm{Au}$ antigen of 1 in $153(0.653 \%)$ in men prisoners has no obvious explanation. Viral hepatitis is not a serious clinical problem in the two institutions concerned, and the positive donors are not drug addicts. What is not known is whether or not these men were Au positive at the time of their first imprisonment. The high incidence may be related to social habits and to hygiene.

A higher incidence of $\mathrm{Au}$ antigen in men compared with women donors has been described (Banke et al., 1971), though unlike the present series the difference was not statistically significant. Differences in incidence are unlikely to be genetically determined (Cossart, 1971), and it is suggested that the difference in incidences in the sexes may again be related to social habits and hygiene. The higher incidence of antibody in women compared with non-prisoner men shown in Table II is not statistically significant, but a similar observation has been made in Danish donors (Banke et al., 1971). This point should therefore be investigated in larger series.

It would be wrong to cause unnecessary worry among the families of donors found to be positive for either $\mathrm{Au}$ antigen or its antibody. Since volunteers have for many years past been asked questions about jaundice, and recent publicity has highlighted the transmission of viral hepatitis by transfusion, it has not been difficult to explain to positive donors that they should refrain from donating blood in the meantime. They have also been easily persuaded to consult their general practitioners. Initially an attempt was made to study other members of the household for $\mathrm{Au}$ antigen and its antibody. In one family studied the wife of an Au-antigen-positive donor was found to have the antibody. Soon afterwards it became clear, however, that family studies might cause alarm, and these have been discontinued. From information received from general practitioners hepatitis has not been an overt illness in the households of positive donors. It seems that these donors have not been a danger to household contacts, and if the infection is transmitted the illness is mild or asymptomatic.

We thank the donors and their medical practitioners for willing co-operation. This study would have been impossible without the loyal support of our technical staff.

\section{References}

Banke, O., Dybkjaer, E., Nordenfelt, E., and Reinicke, V. (1971). Lancet, 1,860 Bulletin of the World Health Organization, 1970, 42, 957. Cossart, Y. E. (1971). Fournal of Clinical Pathology, 24, 394 Kohn, J., and Morgan, J. R. (1971). Fournal of Clinical Pathology, 24, 673. Milne, G. R., and Barr, A. (1971). Sterilin Technical Bulletin, No. 1. Milne, G. R., Barr, A., and Wallace, J. (1971). Lancet, 1, 77. Wallace, J. (1970). Lancet, 2, 609. 